\title{
Novel GaAs Metal-Semiconductor Field-Effect Transistors with InGaP/GaAs Multiple Quantum Barrier Capping and Buffer Layers
}

\author{
Ching-Ting LEE*, Hsin-Ying LEE and Hao-Hsiung LIN ${ }^{1}$ \\ Institute of Optical Sciences, National Central University, Chung-Li, Taiwan, R.O.C. \\ ${ }^{1}$ Department of Electrical Engineering, National Taiwan University, Taipei, Taiwan, R.O.C.
}

(Received March 28, 2002; revised manuscript received June 28, 2002; accepted for publication July 4, 2002)

We present a novel GaAs metal-semiconductor field-effect transistor (MESFET) with InGaP/GaAs multiple quantum barrier (MQB) capping and buffer layers. The MQB structure with $(29 ; 6,5,7 ; 8,8,1 ; 6,5,7 ; 29)$ periodic stacks was designed to increase effective-potential-barrier height. We demonstrate that, by using the InGaP/GaAs MQB capping layer, the gate Schottky barrier performance and gate leakage current are improved. [DOI: 10.1143/JJAP.41.5937]

KEYWORDS: GaAs MESFETs, InGaP/GaAs multiple quantum barrier, Schottky barrier, semiconductor device fabrication

\section{Introduction}

To increase the device density and operational speed of microwave and electrooptical integrated circuits, the parasitic interaction and isolation among active devices have to be significantly reduced and enhanced. To reduce the sidegating effect and parasitic interaction influenced from GaAs substrate-related problems, ${ }^{1,2)}$ various buffer layer structures grown between channel layer and substrate have been developed. ${ }^{3,4)}$ As for the increase in effective-potentialbarrier height using multiple quantum barrier (MQB) structure, ${ }^{5)}$ recently the $\mathrm{AlGaAs} / \mathrm{GaAs} \mathrm{MQB}$ structure was successfully employed as a buffer layer, effectively reducing parasitic interactions and improving isolation. ${ }^{6,7)}$ Compared to the $\mathrm{AlGaAs} / \mathrm{GaAs}$ heterostructure, the $\mathrm{InGaP} / \mathrm{GaAs}$ heterostructure has a larger band discontinuity ${ }^{8)}$ and a high selective etching rate between $\mathrm{InGaP}$ and $\mathrm{GaAs} .{ }^{9)}$ To reduce sidegating effect and improve isolation due to the increase of effective-potential-barrier height, we demonstrated the InGaP/GaAs MQB structure, instead of AlGaAs/GaAs MQB structure, grown between GaAs channel layer and substrate as a buffer layer in GaAs metal-semiconductor field-effect transistor (MESFET), recently. ${ }^{10)}$ In this paper, we employed the InGaP/GaAs MQB structure for the capping and buffer layers in the GaAs MESFET configuration. The InGaP/GaAs MQB capping layer was used to improve Schottky barrier performance and reduce gate leakage current of the GaAs MESFET. At the same time, to maintain the superior isolation and good carrier configuration, an InGaP/GaAs MQB buffer layer was used in the MESFET's structure.

\section{Device Structure and Fabrication Process}

The epitaxial layers and MQB structure used in the GaAs MESFET configuration shown in Fig. 1 were grown using a gas-source molecular beam epitaxial system (GSMBE). The solid sources employed were In, Ga and $\mathrm{Si}$; while the gas sources of $\mathrm{As}$ and $\mathrm{P}$ were $\mathrm{AsH}_{3}$ and $\mathrm{PH}_{3}$, respectively. After first growing an undoped GaAs $(300 \mathrm{~nm})$ buffer layer on a (100) semi-insulating GaAs substrate, a second, undoped InGaP/GaAs MQB buffer layer, as well as a Si-doped GaAs channel layer $\left(300 \mathrm{~nm}, 3 \times 10^{17} \mathrm{~cm}^{-3}\right)$, undoped $\mathrm{InGaP} /$ GaAs MQB capping layer, undoped InGaP Schottky enhancement layer $(20 \mathrm{~nm})$, and a heavily Si-doped $\mathrm{n}^{+}$-

*Corresponding author. E-mail: t260003@cc.ncu.edu.tw

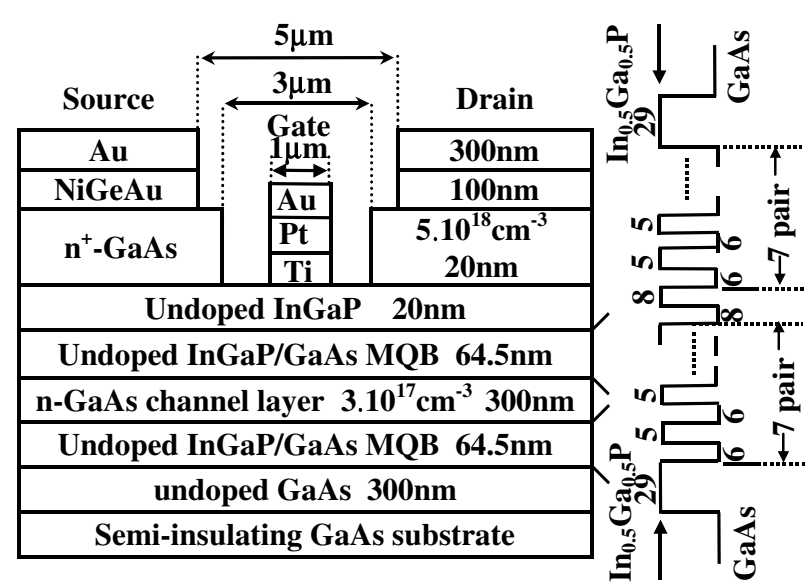

Fig. 1. Epitaxial structure and schematic diagram of InGaP/GaAs MQB structure in monolayer of GaAs MESFET.

GaAs ohmic contact layer $\left(20 \mathrm{~nm}, 5 \times 10^{18} \mathrm{~cm}^{-3}\right)$, were all grown sequentially. Because superior Schottky barrier performances of the Ti/Pt/Au metallization system contacted with the wide-bandgap InGaP material were reported previously. ${ }^{11)}$ To obtain high Schottky barrier height performance, an undoped InGaP layer was grown on the top of the InGaP/GaAs MQB capping layer as well. Furthermore, to achieve good ohmic performances for the MESFET's source and drain regions, a thin $\mathrm{n}^{+}-\mathrm{GaAs}$ was grown on the undoped InGaP Schottky enhancement layer. The InGaP/GaAs MQB buffer and capping layers were designed with the same structure. This MQB structure, with three periodic stacks, is described as $\left(B_{0} ; W_{1}, B_{1}, P_{1} ; W_{2}, B_{2}\right.$, $\left.P_{2} ; W_{3}, B_{3}, P_{3} ; B_{0}\right)$, where $B_{0}$ is the number of monolayer of the InGaP barrier grown on the top and bottom regions of the InGaP/GaAs MQB structure; $W_{i}$, and $B_{i},(i=1,2,3)$ are the number of monolayer of the following GaAs well and InGaP barrier, respectively; $\mathrm{P}_{i},(i=1,2,3)$ is the pair number of the GaAs well and InGaP barrier. The InGaP/GaAs MQB structure designed for the MESFETs was constructed by (29; $6,5,7 ; 8,8,1 ; 6,5,7 ; 29)$. There was a total of 228 monolayers and the MQB structure's thickness was approximately $64.5 \mathrm{~nm}$. To evade low-energy electron tunneling, a thicker InGaP barrier, with 29 monolayers, was grown on the top and bottom regions of the MQB structure. The schematic diagram of the InGaP/GaAs MQB structure is shown in Fig. 1. To investigate the epitaxial 


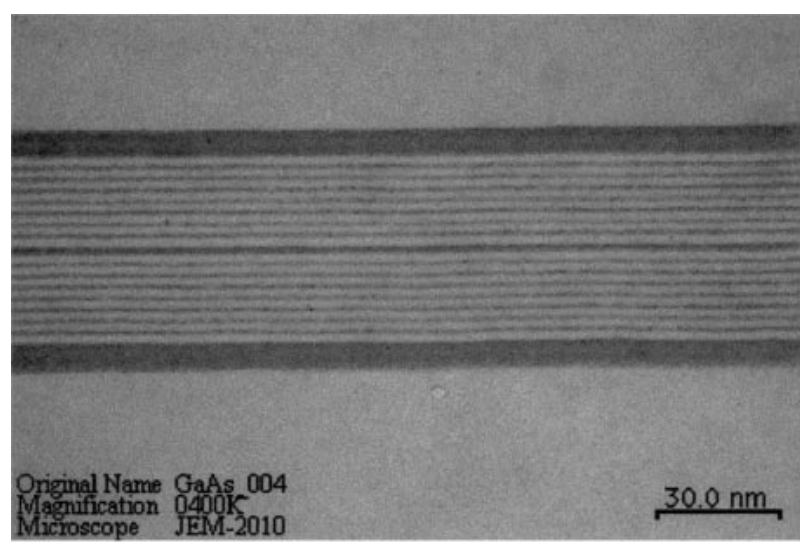

(a)

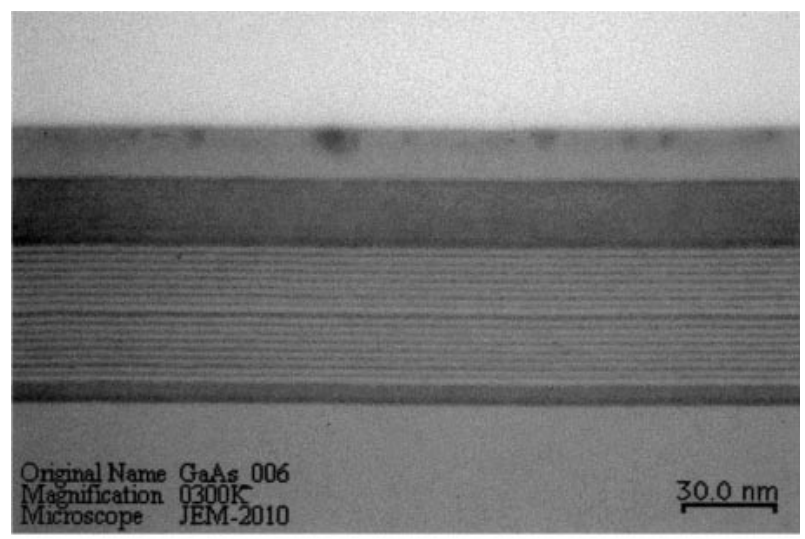

(b)

Fig. 2. TEM micrograph of (a) InGaP/GaAs MQB buffer layer and (b) capping layer.

quality, the InGaP/GaAs MQB structures were examined using a transmission electron microscopy (TEM). The TEM micrograph of the InGaP/GaAs MQB buffer and capping structures is shown in Figs. 2(a) and 2(b), respectively. Here it can be seen that the interface between the InGaP barrier and GaAs well is very flat and sharp. Since samples have to be thermally treated at $400^{\circ} \mathrm{C}$ for 3 min during the ohmic alloy process for source and drain fabrication, the crystal structure of the thermally treated $\mathrm{InGaP} / \mathrm{GaAs} \mathrm{MQB}$ layers should be investigated. The InGaP/GaAs MQB buffer and capping layers treated with temperature at $420^{\circ} \mathrm{C}$ for $30 \mathrm{~min}$ were investigated using TEM. The TEM micrographes reveal the same structure as the as-grown InGaP/GaAs MQB structure shown in Figs. 2(a) and 2(b). No intermixing or deterioration between the InGaP barrier and $\mathrm{GaAs}$ well were found. By using the transfer matrix method, ${ }^{12)}$ the electron reflectivity of the designed $\mathrm{InGaP} / \mathrm{GaAs} \mathrm{MQB}$ structure as a function of the electron energy normalized with $\Delta E_{\mathrm{c}}$ is shown in Fig. 3, where $\Delta E_{\mathrm{c}}=223 \mathrm{meV}$ is the conduction band discontinuity between an undoped InGaP and a GaAs heterostructure. It can be seen that the effective-potentialbarrier height of this designed InGaP/GaAs MQB structure is estimated to be $3.2 \Delta E_{\mathrm{c}}$. In other words, the effectivepotential-barrier height of electron for the designed $\mathrm{InGaP} /$ GaAs MQB structure has been increased to 3.2 times that of the conventional InGaP/GaAs heterostructure. Therefore,

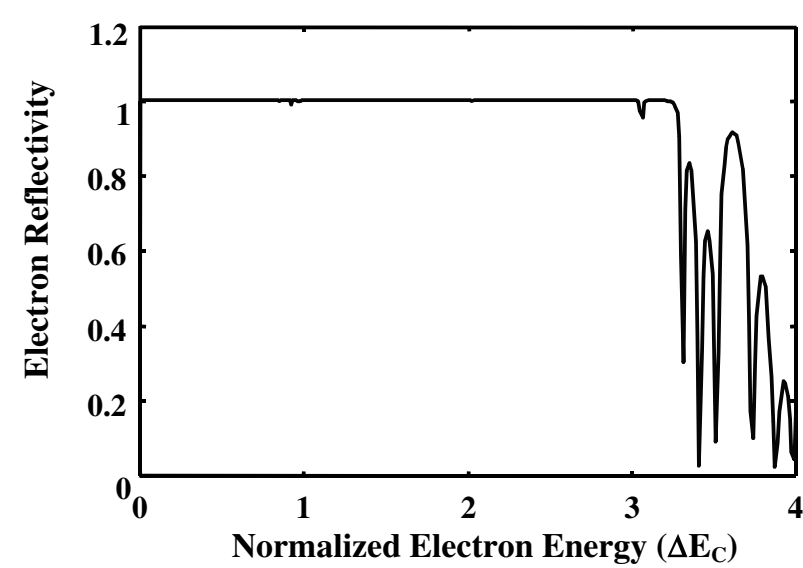

Fig. 3. Electron reflectivity as a function of normalized electron energy of InGaP/GaAs MQB structure.

superior isolation of the designed InGaP/GaAs MQB structure can be expected. In the simulation shown in Fig. 3, we assume the conduction band offset $\Delta E_{\mathrm{c}}=$ $223 \mathrm{meV}$ for a conventional InGaP/GaAs heterostructure, the electron effective mass of InGaP and GaAs is $0.11 m_{0}$ and $0.067 m_{0}$, respectively, ${ }^{13,14)}$ where $m_{0}$ is the electron rest mass.

By using a standard photolithographic technique, the patterns of the source and drain regions for MESFETs were defined. The ohmic metals of NiGeAu/Au $(100 / 300 \mathrm{~nm})$ were evaporated using an electron beam evaporator under a high vacuum environment $\left(1 \times 10^{-6}\right.$ Torr $)$. After using liftoff process, the ohmic contact of the source and drain regions for the MESFETs was thermally alloyed at $400^{\circ} \mathrm{C}$ for $3 \mathrm{~min}$. For the first kind of MESFETs (referred to as FET1), the traditional gate recess was etched down to the GaAs channel layer; while for the second kind of MESFETs (referred to as FET2), the gate recess was performed by only etching the $\mathrm{n}^{+}-\mathrm{GaAs}$ ohmic contact layer, and stopping at the undoped InGaP Schottky enhancement layer by selective etching solution of $\mathrm{H}_{2} \mathrm{SO}_{4}: \mathrm{H}_{2} \mathrm{O}_{2}: \mathrm{H}_{2} \mathrm{O}(1: 8: 80)$. Following the gate recess process, a gate pattern with a length of $1 \mu \mathrm{m}$ and a width of $25 \mu \mathrm{m}$ was defined and aligned in the photoresist. The gate metals of Ti/Pt/Au (50/100/300 nm) were then evaporated and the excess metals were removed using the lift-off technique.

\section{Experimental Results and Discussion}

The output characteristics of the drain-source current $\left(I_{\mathrm{ds}}\right)$ as a function of the drain-source voltage $\left(V_{\mathrm{ds}}\right)$ for the FET2 is shown in Fig. 4. The $I_{\mathrm{dss}}$ value of the drain-source current obtained in saturation with gate-source voltage $V_{\mathrm{gs}}=0$ is $225 \mathrm{~mA} / \mathrm{mm}$. The $I_{\mathrm{ds}}$ value at $V_{\mathrm{gs}}=1 \mathrm{~V}$ is $272 \mathrm{~mA} / \mathrm{mm}$. The pinch-off voltage $V_{\mathrm{p}}$ is $-7.5 \mathrm{~V}$. The $I_{\mathrm{dss}}$ value and pinch-off voltage $V_{\mathrm{p}}$ for the FET1 are $138 \mathrm{~mA} / \mathrm{mm}$ and $-2.5 \mathrm{~V}$, respectively. The discrepancy of the $I_{\mathrm{dss}}$ and $V_{\mathrm{p}}$ between the FET2 and FET $1{ }^{10)}$ resulted from the different recess depth of the gate region. The MESFETs exhibited complete pinch-off phenomena at associated pinch-off voltage. This phenomena show that the InGaP/GaAs MQB buffer layer has a very low carrier concentration and the interface between the GaAs channel layer and the MQB buffer possesses very good crystal quality. Furthermore, superior isolation between the 


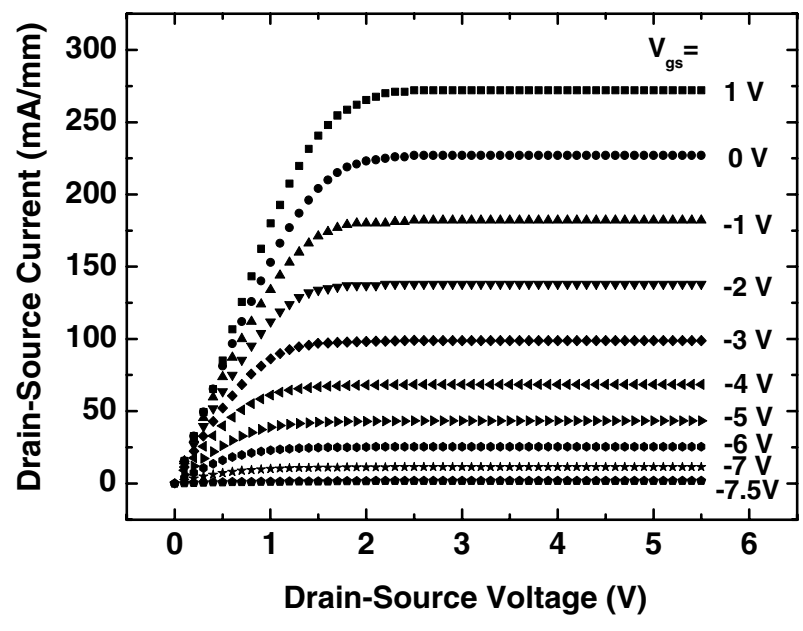

Fig. 4. Typical output characteristics of drain-source current as a function of drain-source voltage for FET2.

GaAs channel layer and the MQB buffer layer is expected due to the increase in effective-potential-barrier height of the InGaP/GaAs MQB buffer layer.

To investigate the function of the InGaP/GaAs MQB capping layer, the first of these is to measure the forward $I-$ $V$ characteristics of the gate at room temperature. Figure 5 shows the gate forward bias voltage $V_{\mathrm{gs}}$ versus gate current $I_{\mathrm{gs}}$. The enlarge current range is shown in the inset of Fig. 5. According to the thermionic emission theory, the Schottky barrier height of $1.06 \mathrm{eV}$ and ideality factor of 1.19 can be obtained. Figure 6 shows the dependence of the gate-source current $\left(I_{\mathrm{gs}}\right)$ as a function of the reverse gate-source voltage $\left(V_{\mathrm{gs}}\right)$ at floating drain, and $V_{\mathrm{ds}}=2$ and $3 \mathrm{~V}$ for FET1 and FET2. When the drain is floating, it can be seen that the gate breakdown voltage for FET1 and FET2 is 12.5 and $22 \mathrm{~V}$, respectively. The breakdown voltage is defined as the knee voltage of the $I_{\mathrm{gs}}-V_{\mathrm{gs}}$ characteristics. According to this experimental result, when the drain-source current passes through the region under gate region by applying drainsource voltage, the gate leakage current $\left(I_{\mathrm{gs}}\right)$ for FET2 is significantly lower than that of FET1. If the gate leakage current of $10 \mathrm{nA}$ can be accepted tolerantly, the gate-source

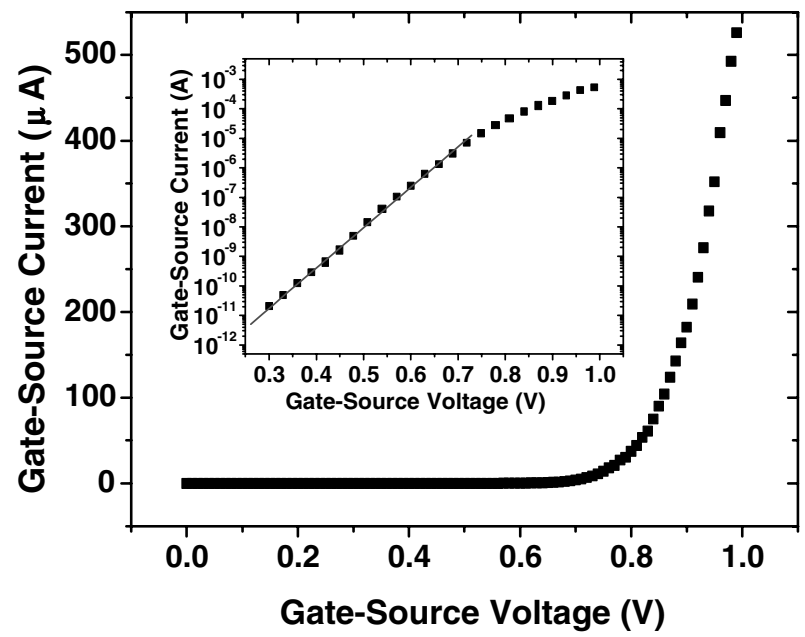

Fig. 5. Typical characteristics of gate forward bias voltage $V_{\mathrm{gs}}$ versus gate current $I_{\mathrm{gs}}$.

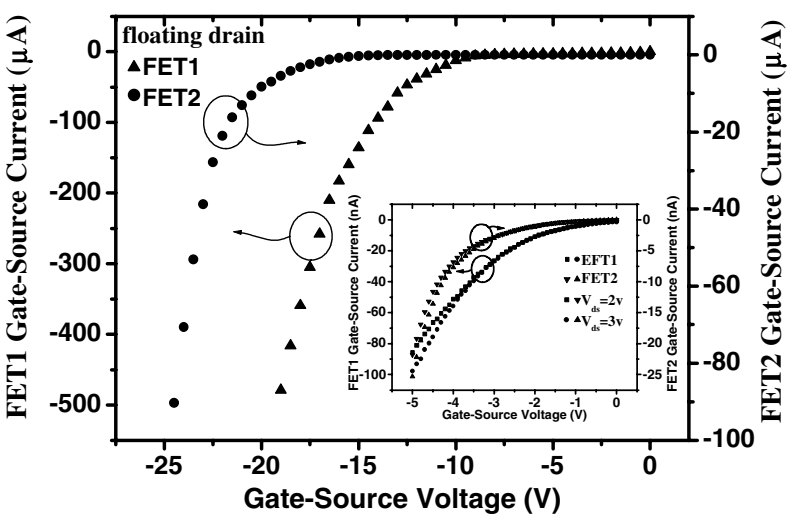

Fig. 6. Typical characteristics of gate-source current as a function of the reverse gate-source voltage for floating drain and $V_{\mathrm{ds}}=2$ and $3 \mathrm{~V}$ of FET1 and FET2.

voltage for FET1 and FET2, operated at $V_{\mathrm{ds}}=3 \mathrm{~V}$, is -1.8 and $-4.2 \mathrm{~V}$, respectively. In the FET1 structure, the gate leakage current flows through the GaAs channel layer to the $\mathrm{Ti} / \mathrm{Pt} / \mathrm{Au}$ Schottky metals. In the FET2 structure, the gate leakage current flows through the GaAs channel layer, InGaP/GaAs MQB cap layer, InGaP Schottky enhancement layer, and Ti/Pt/Au Schottky metals. The gate leakage current of FET2 is far superior to that of FET1, not only because the Schottky performance of the Ti/Pt/Au contacted with $\mathrm{InGaP}$ is better than that of $\mathrm{Ti} / \mathrm{Pt} / \mathrm{Au}$ contacted with GaAs, ${ }^{11)}$ but also because the increase of the effectivepotential-barrier height and the evasion of low-energy electron tunneling are designed in the $\mathrm{InGaP} / \mathrm{GaAs} \mathrm{MQB}$ capping layer.

To study thermal stability, a thermal test was carried out on both FET1 and FET2. Figure 7 shows typical characteristics of $I_{\mathrm{gs}}$ versus $V_{\mathrm{gs}}$ operated at $V_{\mathrm{ds}}=3 \mathrm{~V}$, for the FET1 and FET2, after thermal treatment at various temperatures for $30 \mathrm{~min}$, respectively. It can be seen that the gate leakage current increases slowly with temperature for FET2; whereas it increases dramatically for FET1. This gate leakage current phenomenon is attributable to the dependence of the thermionic emission current on temperature. Since the crystal structure of the $\mathrm{InGaP} / \mathrm{GaAs}$ MQB capping layer can be protected during the ohmic alloy process for the

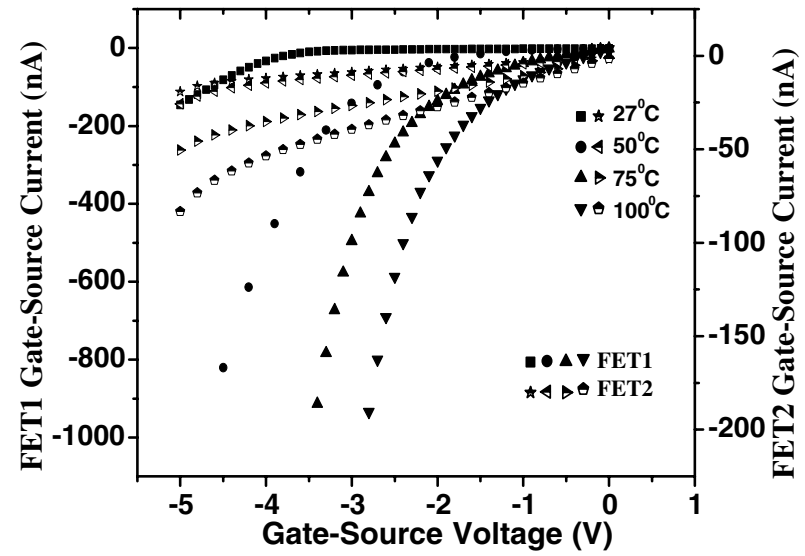

Fig. 7. Typical characteristics of gate-source current versus gate-source voltage at $V_{\mathrm{ds}}=3 \mathrm{~V}$ thermally treated at various temperatures for $30 \mathrm{~min}$ of FET1 and FET2. 
source and drain fabrication, and the thermal test mentioned above, the increase in the effective-potential-barrier height for the MQB structure is preserved. Due to the reported result that the Schottky barrier height for the Ti/Pt/Au metallization system contacted with $\mathrm{InGaP}$ is superior to $\mathrm{Ti} /$ $\mathrm{Pt} / \mathrm{Au}$ directly contacted with $\mathrm{GaAs},{ }^{11)}$ and because of the increase in the effective-potential-barrier height of the InGaP/GaAs MQB capping layer, the gate leakage current for FET2 increases much more slowly with temperature than that of FET1.

\section{Conclusion}

This paper proposed and fabricated a novel MESFET configuration with $\mathrm{InGaP} / \mathrm{GaAs} \mathrm{MQB}$ buffer and capping layers. For the designed $\mathrm{InGaP} / \mathrm{GaAs} \mathrm{MQB}$ structure with $(29 ; 6,5,7 ; 8,8,1 ; 6,5,7 ; 29)$ periodic stacks, the effectivepotential-barrier height was increased to 3.2 times of the conduction band discontinuity between an undoped InGaP and GaAs heterostructure. Gate leakage was significantly reduced and thermal stability was significantly improved for the novel MESFETs through use of an InGaP/GaAs capping layer and InGaP Schottky enhancement layer. The gate breakdown voltage of the $\mathrm{InGaP} / \mathrm{GaAs} \mathrm{MQB}$ capping layer with and without the undoped InGaP layer is 22 and $17 \mathrm{~V}$, respectively, while the gate breakdown voltage without both the InGaP/GaAs MQB capping layer and the undoped InGaP layer is $12.5 \mathrm{~V}$. We can conclude that the dominant function of the $\mathrm{InGaP} / \mathrm{GaAs} \mathrm{MQB}$ capping layer is to isolate the gate leakage, and the dominant function of the undoped InGaP layer is to enhance the Schottky barrier height. In conclusion, according to the experimental results, this proposed novel MESFET configuration with InGaP/GaAs MQB buffer and capping layers is a promising structure for future high density integrated circuit designs and applications.

\section{Acknowledgement}

This work was supported from the National Science Council of the Republic of China.

1) C. Kocot and C. A. Stolte: IEEE Trans. Microwave Theory Tech. 30 (1982) 963.

2) K. Horio and K. Satoh: IEEE Trans. Electron Devices 41 (1994) 2256.

3) C. L. Ghosh and R. L. Layman: IEEE Electron Device Lett. 5 (1984) 3.

4) F. W. Smith, A. R. Calawa, C. L. Chen, M. J. Manfra and L. J. Mahoney: IEEE Electron Device Lett. 9 (1988) 70.

5) T. Takagi, F. Koyama and K. Iga: Jpn. J. Appl. Phys. 29 (1990) 1977.

6) C. T. Lee, C. D. Tsai, C. Y. Wang, H. P. Shiao, T. E. Nee and J. N. Shen: Appl. Phys. Lett. 67 (1995) 2046.

7) C. T. Lee: IEEE Trans. Electron Devices 45 (1998) 2083.

8) D. Biswas, N. Debbar, P. Bhattacharya, M. Razeghi, M. Defour and F. Omnes: Appl. Phys. Lett. 56 (1990) 833.

9) J. R. Lothian, J. M. Kuo, F. Ren and S. J. Pearton: J. Electron. Mater. 21 (1992) 441.

10) C. T. Lee, K. C. Shyu, I. J. Lin and H. H. Lin: Mater. Sci. Eng. B 74 (2000) 147.

11) C. T. Lee, H. P. Shiao, N. T. Yeh, C. D. Tsai, Y. T. Lyu and Y. K. Tu: Solid State Electron. 41 (1997) 1.

12) B. Jonsson and S. T. Eng: IEEE J. Quantum Electron. 26 (1990) 2025.

13) S. Obrien, J. R. Shealy and D. P. Bour: Appl. Phys. Lett. 53 (1988) 1859.

14) V. Swaminathan and A. T. Macrander: Materials Aspects of GaAs and InP Based Structures (Prentice-Hall, New Jersey, 1991) p. 19. 